\title{
Фотоуправляемые возвратно-поступательные молекулярные машины типа "гость-хозяин"
}

\author{
В. М. Розенбаум ${ }^{+1)}$, М. Л. Дехтярь , И. В. Шапочкина ${ }^{\times}$, Л. И. Трахтенберг ${ }^{\circ}$ \\ + Институт химии поверхности им. А. А. Чуйко НАН Украины, 03164 Киев, Украина \\ ${ }^{*}$ Институт органической химии НАН Украины, 02660 Киев, Украина \\ × Белорусский государственный университет, физический факультет, 220050 Минск, Беларусь \\ ○ Федеральный исследовательский центр химической физики им. Н. Н. Семенова РАН, 119991 Москва, Россия

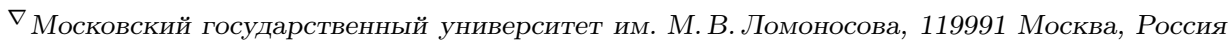 \\ Поступила в редакцию 21 апреля 2021 г. \\ После переработки 26 апреля 2021 г. \\ Принята к публикации 26 апреля 2021 г.
}

\begin{abstract}
Установлены общие принципы функционирования управляемых светом молекулярных машин (lightdriven molecular machines) с различными потенциальными энергиями броуновской частицы в силовом поле окружения в основном и возбужденном состояниях. Оптико-механическая связь временных зависимостей заселенности фотовозбужденного состояния частицы и ее координаты описывается в рамках теории, построенной для возвратно-поступательного броуновского фотомотора, состоящего из молекул красителя-гостя и кавитанда-хозяина и приводимого в действие лазерными импульсами. Рассчитаны временные зависимости спектроскопических и механических характеристик в приближении параболических потенциальных профилей основного и возбужденного состояний. Показано, что зависимость средней скорости возвратно-поступательного движения от периода следования сверхкоротких лазерных импульсов является немонотонной функцией с максимумом, определяющим оптимальный режим работы молекулярной машины. В случае, когда втягивание молекулы красителя в полость кавитанда затрудняет образование тушащего флуоресценцию скрученного состояния и константа скорости дезактивации молекулы-гостя убывает с ее координатой, время жизни флуоресценции полностью втянутого в кавитанд красителя существенно увеличивается, в согласии с имеющимися экспериментальными данными. Представленные в работе аналитические соотношения между механическими и оптическими характеристиками рассматриваемых возвратно-поступательных молекулярных машин открывают широкие возможности для управления их движением, а также наблюдаемой для них флуоресценцией.
\end{abstract}

DOI: $10.31857 / \mathrm{S} 1234567821110100$

Принципы действия наномашин, преобразующих различные виды неравновесных флуктуаций в направленное движение, обычно описываются соответствующими моделями броуновских рэтчетов. В таких моделях подразумевается, что поступление энергии в систему характеризуется временной зависимостью потенциальной энергии $U(x, t)$ броуновской частицы, взаимодействующей с окружением $(x=$ $=x(t)-$ координата частицы, $t-$ время). Эта временная зависимость может возникать вследствие различных процессов: конформационных переходов под действием протекающих в частицах химических реакций (как в белковых молекулярных моторах), переключения электрических полей между электродами, изменения температуры системы, интерферен-

\footnotetext{
1) e-mail: vik-roz@mail.ru
}

ции лазерных пучков или лазерных импульсов, вызывающих электронные переходы в частицах [1-4]. Важную роль в функционировании броуновских рэтчетов играют также симметрийные закономерности $[5,6]$.

Если направленное (поступательное, вращательное или возвратно-поступательное) движение наночастиц инициируется их фотовозбуждением, то говорят о броуновских фотомоторах [7-10]. Такие наноустройства характеризуются различными распределениями плотности заряда частицы (молекулы) в основном $\mathrm{S}_{0}$ и возбужденном $\mathrm{S}_{1}$ электронных состояниях и, следовательно, различными соответствующими потенциальными энергиями $U_{0}(x)$ и $U_{1}(x)$. Анализ координатных зависимостей потенциальных профилей $U_{0}(x)$ и $U_{1}(x)$ позволяет наглядно объяснить принципы работы поступа- 
тельных [10] и вращательных [11] фотомоторов. Если плотность вероятности нахождения частицы с координатой в состоянии $\mathrm{S}_{j}(j=0,1)$ в момент времени $t$ обозначить через $\rho_{j}(x, t)$, то средняя потенциальная энергия определяется соотношением $U(x, t)=\sum_{j} \rho_{j}(x, t) U_{j}(x)$ (приближенно можно считать, что $U(x, t)=\sum_{j} n_{j}(t) U_{j}(x)$ [10], где $n_{j}(t)=\int \rho_{j}(x, t) d x-$ заселенность состояния $\mathrm{S}_{j}$ ). Часто также рассматривается пространственно-временная зависимость потенциальной энергии аддитивно-мультипликативного вида $U(x, t)=u(x)+\sigma(t) w(x)[12,13]$, в котором $u(x)$ и $w(x)$ - произвольные функции координаты, а функция $\sigma(t)= \pm 1$ описывает детерминистический или стохастический дихотомный процесс. В данной статье мы представляем общую схему описания фотомоторов, управляемых периодически следующими мгновенными лазерными импульсами, причем такой подход не требует введения средней потенциальной энергии.

Управляющим уравнением для функций распределения $\rho_{j}(x, t)$, описывающих диффузионную динамику и перераспределение частиц между состояниями $\mathrm{S}_{j}$, является уравнение Смолуховского с источниками и стоками [14]:

$$
\frac{\partial \rho_{j}(x, t)}{\partial t}=-\frac{\partial J_{j}(x, t)}{\partial x}+(-1)^{j} \Gamma(x) \rho_{1}(x, t), \quad j=0,1,
$$

где

$$
\begin{gathered}
J_{j}(x, t)=-D_{j} e^{-\beta U_{j}(x)} \frac{\partial}{\partial x} e^{\beta U_{j}(x)} \rho_{j}(x, t)= \\
=-D_{j} \frac{\partial}{\partial x} \rho_{j}(x, t)-\zeta_{j}^{-1} U_{j}^{\prime}(x) \rho_{j}(x, t)
\end{gathered}
$$

- поток частиц $\left(U_{j}^{\prime}(x)=d U_{j}(x) / d x, \zeta_{j}\right.$ - коэффициент трения в состоянии $\mathrm{S}_{j}, D_{j}=k_{B} T / \zeta_{j}$ - соответствующий коэффициент диффузии, $\beta=\left(k_{B} T\right)^{-1}$, $k_{B}$ - постоянная Больцмана, $T$ - абсолютная температура), а $\Gamma(x)$ - полная константа скорости перехода из возбужденного электронного состояния в основное (учитывающая как излучательные, так и безызлучательные процессы), которая в общем случае может зависеть от координаты частицы (рис. 1).

Выведем уравнения для первых моментов распределений, а именно, заселенностей $n_{j}(t)=\int \rho_{j}(x, t) d x$ и средних значений координаты $\langle x(t)\rangle_{j}=\int x \rho_{j}(x, t) d x$ в состояниях $\mathrm{S}_{j}$ (область интегрирования зависит от типа рассматриваемого движения). Уравнение для $n_{j}(t)$ получается путем почленного интегрирования уравнения (1) по периоду поступательного или вращательного движения, или же по всей области изменения координаты,

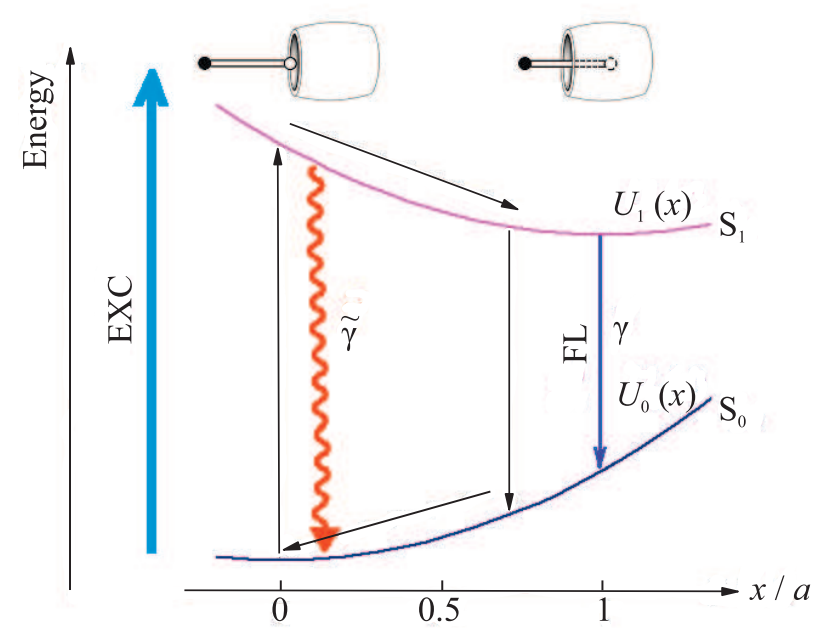

Рис. 1. (Цветной онлайн) Схема функционирования управляемой светом возвратно-поступательной молекулярной машины типа "гость-хозяин”, состоящей из молекул красителя и кавитанда. Под действием сверхкороткого лазерного импульса молекула красителя переходит во франк-кондоновское состояние, а затем релаксирует в возбужденное состояние с потенциальным профилем, обеспечивающим полное втягивание молекулы красителя в полость кавитанда. После дезактивации возбужденного состояния, вызванной излучательными и безызлучательными переходами в основное состояние, молекула красителя возвращается в начальное (частично втянутое в кавитанд) положение.

описывающей возвратно-поступательное движение. Уравнение для $\langle x(t)\rangle_{j}$ получается аналогично, но с почленным домножением (1) на $x$ и последующим использованием интегрирования по частям. В случае поступательного или вращательного движения интегралы от производных периодических функций по периоду равны нулю, тогда как для возвратнопоступательного движения они обращаются в нуль вследствие обращения в нуль функций распределения и потоков на границах рассматриваемой области $\left(U_{j}( \pm \infty) \rightarrow 0, U_{j}(x)\right.$ - удерживающие потенциалы). В результате получаем следующие уравнения, справедливые для всех вышеуказанных типов движения броуновского фотомотора:

$$
\begin{gathered}
\frac{d n_{j}(t)}{d t}=(-1)^{j}\langle\Gamma(x)\rangle_{1}, \quad \sum_{j} n_{j}(t)=1, \\
\frac{d\langle x(t)\rangle_{j}}{d t}=-\zeta_{j}\left\langle U_{j}^{\prime}(x)\right\rangle_{j}+(-1)^{j}\langle x \Gamma(x)\rangle_{1} .
\end{gathered}
$$

Для рассматриваемого в данной статье возвратно-поступательного движения потенциальные энергии $U_{j}(x)$ соответствуют удерживающим потенциалам, форма которых определяется рас- 
сматриваемым объектом. Например, для полимеров, состоящих из бистабильных фоточувствительных азобензольных звеньев, можно чередовать удлиненное и сжатое состояния, переключая лазерными импульсами транс- и цис-конфигурации азогрупп [15]. При этом потенциальный профиль $U_{0}(x)-$ двухъямный (бистабильный), а $U_{1}(x)$ - параболический. Сдвинутыми относительно друг друга параболическими профилями $U_{0}(x)$ и $U_{1}(x)$ описывается возвратно-поступательное движение двух связанных белковых частиц, которое вследствие специфики белкового трения (зависимости коэффициента трения от скорости скольжения) может быть преобразовано в поступательное движение этих частиц вдоль поверхности [16-18].

Если рассматривать потенциальные профили в параболическом приближении $U_{j}(x)=k_{j}\left(x-a_{j}\right)^{2} / 2$, а константу скорости дезактивации считать независящей от координаты, т.е. $\Gamma(x)=\gamma$, то средние значения величин в уравнениях (3) можно выразить через первые моменты распределений $\rho_{j}(x, t):\left\langle U_{j}^{\prime}(x)\right\rangle_{j}=$ $=k_{j}\langle x(t)\rangle_{j}-k_{j} a_{j} n_{j}(t),\langle\Gamma(x)\rangle_{1}=\gamma n_{1}(t),\langle x \Gamma(x)\rangle_{1}=$ $=\gamma\langle x(t)\rangle_{1}$. С учетом этого система уравнений (3) дает замкнутое описание возвратно-поступательного движения. Для простоты будем считать, что кривизна потенциальных рельефов и коэффициенты трения одинаковы в основном и возбужденном состояниях $\left(k_{j}=k, \zeta_{j}=\zeta\right)$, а минимумы парабол в этих состояниях находятся в начале координат $x=0$ и в точке $x=a\left(a_{0}=0, a_{1}=a\right)$, так что возвратнопоступательное движение фотомотора совершается между этими точками (рис. 1).

В современной нанофотонике используются фемтосекундные лазерные импульсы, позволяющие достигать высоких интенсивностей электромагнитного излучения без необратимого разрушения материала и таким образом осуществлять уникальные режимы взаимодействия света с наноструктурами и молекулярными агрегатами [19]. Поскольку движение нанообъектов характеризуется временами порядка пикои наносекунд, то импульсы с длительностями менее 40 фс [20] можно считать практически мгновенными. В момент окончания сверхкороткого лазерного импульса успевает измениться (за $10^{-15}$ с) только распределение электронной плотности молекулы, в то время как положения ядер и, соответственно, положение молекулы в целом остаются неизменными, т.е. молекула переходит во франк-кондоновское возбужденное состояние [21] согласно адиабатическому приближению Борна-Оппенгеймера. Поэтому начальное положение молекулы (со средней координатой $x$ ) после действия импульса остается неизменным, тогда как минимум потенциальной энергии сдвигается в точку $x=a$.

Возвратно-поступательное движение молекулы можно осуществить путем генерации периодических сверхкоротких лазерных импульсов с периодом $\tau$. В таком процессе текущее время $t$ удобно характеризовать номером лазерного импульса $l=1,2, \ldots$ и промежутком времени $\tilde{t}$ после его окончания, так что $t=(l-1) \tau+\tilde{t}$, где $0<\tilde{t}<\tau$. Тогда при сделанных выше допущениях и введении новых обозначений $x_{l}(\tilde{t})=\sum_{j}\langle x(t)\rangle_{j}, n_{l}(\tilde{t})=n_{1}(t)$ из уравнений $(3)$ получается следующая система уравнений для функций $x_{l}(\tilde{t})$ и $n_{l}(\tilde{t})$ :

$$
\left\{\begin{array}{l}
\dot{x}_{l}(\tilde{t})+\kappa x_{l}(\tilde{t})=\kappa a n_{l}(\tilde{t}), \\
\dot{n}_{l}(\tilde{t})+\gamma n_{l}(\tilde{t})=0
\end{array}\right.
$$

(где $\kappa=k / \zeta)$.

Далее зададим начальные условия для системы (4), учитывающие, что молекулярное распределение электронной плотности, как отмечалось выше, подстраивается под лазерный импульс практически мгновенно, в то время как сама молекула не меняет свое положение (значения координаты до и сразу после лазерного импульса совпадают), а при небольших $\tau$ не успевает возвращаться в исходное положение:

$n_{l}(0)=1, \quad x_{1}(0)=0, \quad x_{l}(0)=x_{l-1}(\tau) \quad(l=2,3, \ldots)$.

Тогда $n_{l}(\tilde{t})=\exp (-\gamma \tilde{t})$, а решение системы уравнений $(4)$ для $x_{l}(\tilde{t})$ принимает вид:

$$
x_{l}(\tilde{t})=x_{l}(0) e^{-\kappa \tilde{t}}+\frac{\kappa a}{\kappa-\gamma}\left(e^{-\gamma \tilde{t}}-e^{-\kappa \tilde{t}}\right), 0 \leq \tilde{t} \leq \tau,
$$

$x_{l}(0)=\frac{\kappa a}{\kappa-\gamma}\left(e^{-\gamma \tau}-e^{-\kappa \tau}\right) \frac{1-e^{-\kappa \tau(l-1)}}{1-e^{-\kappa \tau}}, \quad l=1,2, \ldots$

На вставке к рис. 2 изображена зависимость $x_{l}(\tilde{t})$ при $\gamma=50$ ГГц и $\kappa=25,250$ ГГц для первых пяти импульсов с интервалом следования $\tau=30$ пс. Эта зависимость имеет переходную область с шириной порядка $\tau+\kappa^{-1}$, в которой функция $x(t)$ изменяется от начального значения $x(0)=0$ до области установившихся значений $(l \gg 1)$, где она становится периодической, $x(t+\tau)=x(t)$. При этом минимальные значения $x_{\min }$, принимаемые функцией $x(t)$, определяются соотношением $(6)$ для $x_{l}(0)$ с $e^{-\kappa \tau(l-1)} \ll 1$, a eе максимальные значения $x_{\max }$ определяются выражениями

$$
x_{\max }\left(\tilde{t}_{\max }\right)=a e^{-\gamma \tilde{t}_{\max }}=a\left(\frac{\kappa}{\gamma} \frac{1-e^{-\gamma \tau}}{1-e^{-\kappa \tau}}\right)^{\gamma /(\gamma-\kappa)},
$$




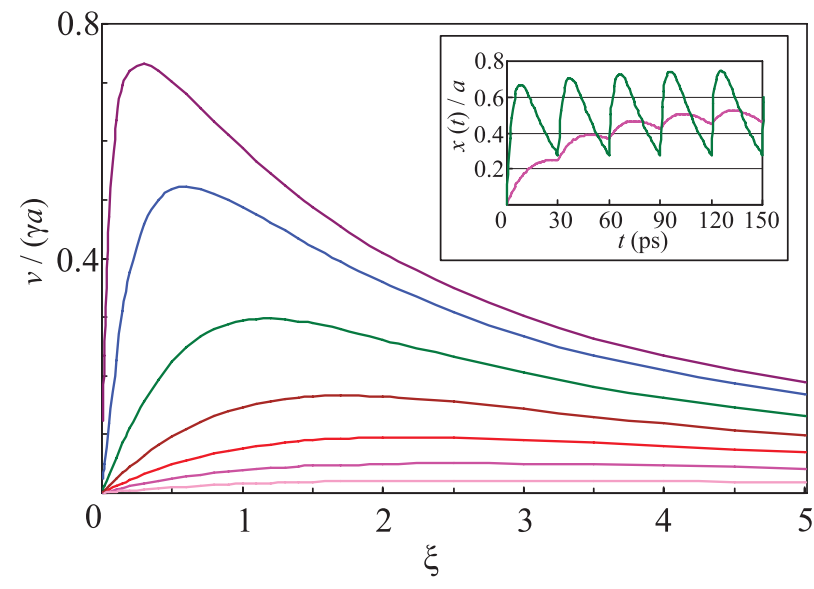

Рис. 2. (Цветной онлайн) Динамика $x(t)$ (на вставке) и средняя скорость возвратно-поступательного движения как функции безразмерного периода $\xi=\gamma \tau$ следования лазерных импульсов, рассчитанные по формулам (6) и (8). Кривые в основной области графика соответствуют снизу вверх $\lambda=\kappa / \gamma=0.2,0.5,1,2,5$, 20, 100. На вставке $\tau=30$ пс, $\gamma=50$ ГГц и $\lambda=0.5,5$ (кривые снизу вверх)

$$
\tilde{t}_{\max }=\frac{1}{\kappa-\gamma} \ln \left(\frac{\kappa}{\gamma} \frac{1-e^{-\gamma \tau}}{1-e^{-\kappa \tau}}\right),
$$

откуда следует аналитическое представление для средней скорости возвратно-поступательного движения:

$$
\begin{gathered}
v \equiv \frac{x_{\max }-x_{\min }}{\tau}= \\
=\frac{\gamma a}{\xi}\left[\left(\lambda \frac{1-e^{-\xi}}{1-e^{-\lambda \xi}}\right)^{1 /(1-\lambda)}+\frac{\lambda}{1-\lambda} \frac{e^{-\xi}-e^{-\lambda \xi}}{1-e^{-\lambda \xi}}\right] \underset{\lambda \rightarrow 1}{\longrightarrow} \frac{\gamma a}{\xi}\left[e^{-\eta(\xi)}+\eta(\xi)-1\right] \\
\eta(\xi) \equiv 1-\frac{\xi}{e^{\xi}-1}, \quad \lambda \equiv \frac{\kappa}{\gamma}, \quad \xi \equiv \gamma \tau .
\end{gathered}
$$

Зависимость средней скорости от безразмерного периода следования импульсов $\xi$ оказывается немонотонной функцией с максимумом, определяющим оптимальный режим работы возвратнопоступательного броуновского мотора. Заметим, что система уравнений (4) и ее решения (6)-(8) не зависят от температуры. Это полностью согласуется с выводами нашей работы [22], в которой было показано, что если константы скоростей переходов не зависят от координаты, а потенциальные энергии $U_{j}(x)$ на больших расстояниях ведут себя как $x^{\alpha}$, то тепловой шум играет конструктивную $(\alpha>2)$ или деструктивную $(\alpha<2)$ роль, а при $\alpha=2$, т.е. при параболических потенциалах, как в нашем случае, играет нейтральную роль.
Перейдем к рассмотрению экспериментально важного случая, когда константа скорости дезактивации $\Gamma(x)$ зависит от координаты молекулы. Влияние зависимости $\Gamma(x)$ на свойства фотомотора проанализирована на примере супрамолекулярной системы типа "гость-хозяин", которая состоит из молекул органического стирилового красителя и кавитанда кукурбитурила и обладает функциями молекулярной машины [23, 24]. Установлено, что в основном электронном состоянии данного комплекса молекула красителя одним из своих концов частично входит в полость молекулы кавитанда. Такое начальное положение центра масс молекулы красителя относительно кавитанда характеризуется значением координаты $x=0$ (на длинной оси молекулыхозяина), в соответствии с положением минимума параболы $U_{0}(x)$ в точке $a_{0}=0$. В возбужденном состоянии минимум потенциальной энергии $U_{1}(x)$ сдвинут по оси $x$ в точку, соответствующую центру масс молекулы красителя, полностью втянутой в кавитанд, $a_{1}=a$ (рис. 1$)$.

Мгновенный лазерный импульс вызывает ряд процессов разного временного масштаба. Во-первых, вследствие быстрой колебательной релаксации $\left(10^{-12}-10^{-11} \mathrm{c}\right)$ и достаточно быстрой $\left(10^{-11}-10^{-10} \mathrm{c}\right)$ внутренней конверсии происходит распад франк-кондоновского состояния во флуоресцентное возбужденное состояние (уровень $\mathrm{S}_{1}$ на рис. 1). Эти быстрые процессы не входят в наше описание, которое включает только последующую деградацию флуоресцентного состояния (соответственно, временная зависимость заселенности этого состояния описывается чисто убывающей функцией, а экспериментально наблюдаемый начальный участок возрастания этой величины в течение нескольких пикосекунд после импульса не рассматривается). Распад состояния $\mathrm{S}_{1}$ включает медленный $\left(10^{-9}-10^{-7}\right.$ c) излучательный переход в основное состояние с константой скорости $\gamma$ и безызлучательные процессы, среди которых преобладает быстрая $\left(10^{-13}-10^{-11}\right.$ c) релаксация путем ортогонального скручивания в состояние TICT (twisted intermolecular charge transfer state - скрученное возбужденное состояние с переносом заряда [25]) с константой скорости $\tilde{\gamma}$.

Состояние ТICT часто дезактивируется посредством мгновенного безызлучательного перехода молекулы в основное состояние через коническое пересечение состояний $\mathrm{S}_{1}-\mathrm{S}_{0}$ (так называемую фотохимическую воронку [25]). По мере втягивания молекулы красителя под действием кулоновских сил [24] в полость кавитанда она вынуждена принимать те 
конформации, которые оптимально заполняют пространство полости, так что ортогонально скрученное состояние ТICT, ведущее к безызлучательной дезактивации, становится все более затрудненным. Поэтому можно предположить, что квантовый выход флуоресценции (а значит, и ее продолжительность) для молекулы красителя, полностью втянутой в полость кавитанда, будет больше, чем в случае частичного втягивания (только одного конца молекулы) [26]. С учетом этого константу скорости распада возбужденного состояния молекулы красителя $Г(x)$ можно задать для простоты линейно убывающей функцией координаты в интервале $0<x<a$ :

$$
\Gamma(x)= \begin{cases}\gamma+\tilde{\gamma}, & x<0 \\ \gamma+\tilde{\gamma}(1-x / a), & 0<x<a \\ \gamma, & x>a .\end{cases}
$$

Измерение многоэкспоненциального затухания флуоресценции действительно подтверждает, что полное втягивание молекулы стирилового красителя вглубь полости кукурбитурила значительно увеличивает самое продолжительное время флуоресценции: примерно с 50 до 100-200 пс [24]. Эти времена по порядку величины определяют значения введенных в соотношениях (9) параметров $\tilde{\gamma}^{-1}$ и $\gamma^{-1}$, соответственно.

Если период следования лазерных импульсов $\tau$ удовлетворяет условию $\tau \gg \gamma^{-1}>\tilde{\gamma}^{-1}$, то молекула красителя успевает между импульсами возвратиться в исходное положение, и все последующие периоды повторяют первый. Введем нормированные на единицу равновесные функции распределения $\rho_{j}^{(\mathrm{eq})}(x)$, соответствующие потенциальным профилям $U_{j}(x)$ $(j=0,1)$ :

$$
\rho_{j}^{(\mathrm{eq})}(x)=\exp \left(-\beta U_{j}(x)\right) / \int_{-\infty}^{\infty} d x \exp \left(-\beta U_{j}(x)\right) .
$$

Тогда можно выбрать следующие начальные условия для уравнений (1), (2) (соответствующие моменту времени сразу после окончания очередного сверхкороткого лазерного импульса): $\rho_{0}(x, \tau)=\rho_{1}(x, 0)=$ $=\rho_{0}^{(\text {eq })}(x), \rho_{1}(x, \tau)=\rho_{0}(x, 0)=0$. Эти уравнения с заданными начальными условиями были решены численно для получения временных зависимостей заселенности возбужденного состояния и средней координаты молекулы красителя (рис. 3 ).

Зависимости $n_{1}(t)$ и $x(t)$ включают два характерных временных интервала. На первом из них, с продолжительностью порядка $\kappa^{-1}$, функция $n_{1}(t)$ быстро спадает (с коэффициентом затухания порядка $\gamma+\tilde{\gamma})$, а функция $x(t)$ быстро возрастает до максимального значения. Обе функции почти не зависят от
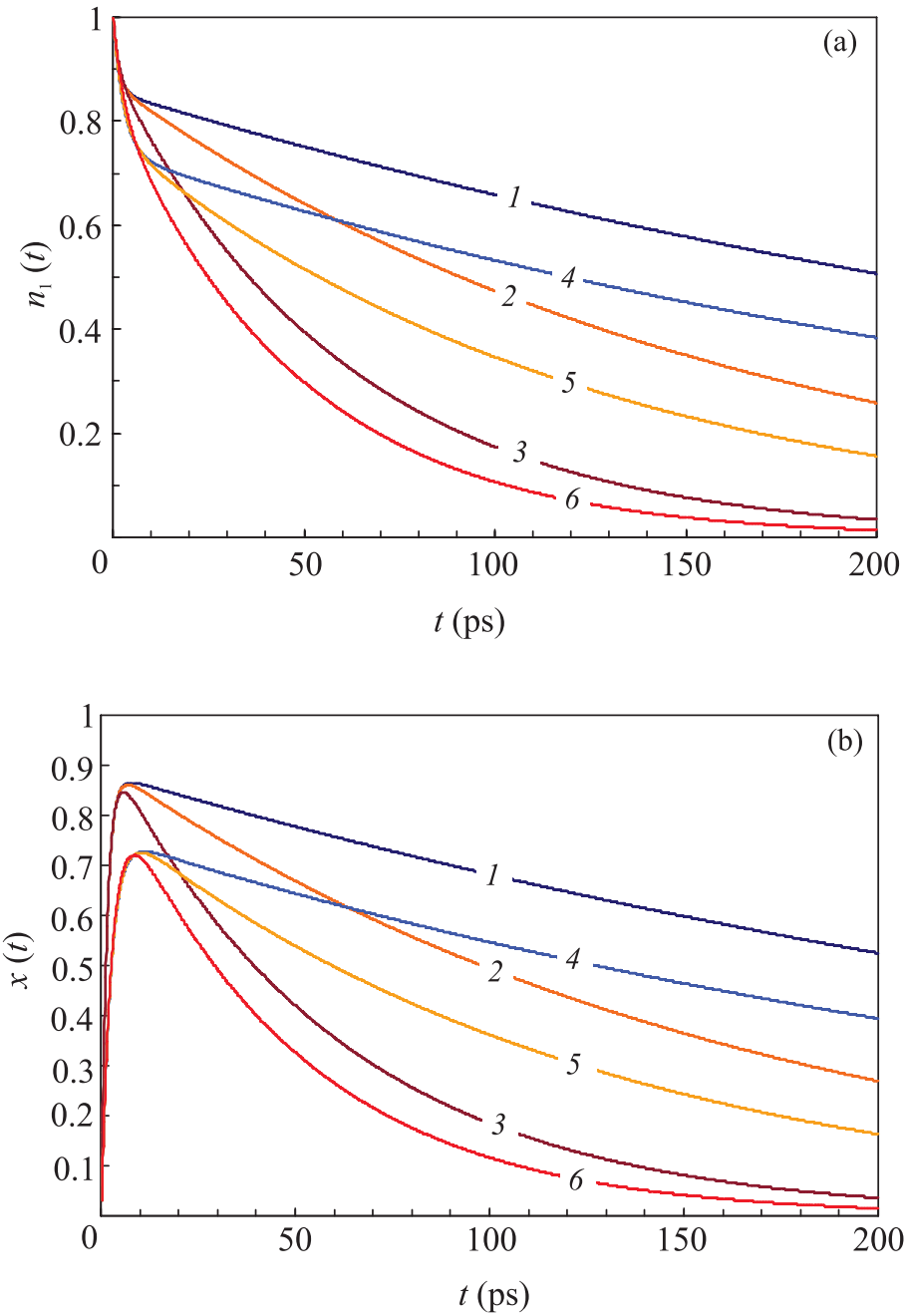

Рис.3. (Цветной онлайн) Временные зависимости заселенности флуоресцентного уровня (a) и координаты (b) молекулы красителя для одного цикла возбуждения при $\tilde{\gamma}=100$ ГГц, $\gamma=1$ ГГц. Кривые 1 -3 соответствуют значениям $D / a^{2}=1,10,100$ Гц с $\kappa=600$ ГГц, а кривые $4-6$ - тем же значениям $D / a^{2}$ с $\kappa=300$ ГГц

величины коэффициента диффузии, что свидетельствует о движении за счет быстрого спуска частицы к минимуму потенциальной ямы в точке $x=a$ (недиффузионная стадия движения). На втором интервале временных зависимостей функция распределения становится пропорциональной $\rho_{1}^{(\mathrm{eq})}(x)$ с коэффициентом пропорциональности $n_{1}(t): \rho_{1}(x, t) \approx$ $\approx n_{1}(t) \rho_{1}^{\text {(eq) }}(x)$ (установление квазиравновесия). Подставляя это распределение в выражение для среднего значения $\langle\Gamma(x)\rangle_{1}$, определяющего скорость ухода системы из возбужденного состояния (см. первое уравнение в $(3))$, получаем $\langle\Gamma(x)\rangle_{1} \approx \bar{\Gamma} n_{1}(t)$, где величина $\bar{\Gamma}$, в дальнейшем называемая коэффициентом затухания, - константа скорости распада возбужден- 
ного состояния на втором временном интервале, на котором функции $n_{1}(t)$ и $x(t)$ медленно спадают до нуля. Если известен потенциальный профиль возбужденного состояния $U_{1}(x)$ (а значит, и функция $\rho_{1}^{(\text {eq) }}(x)$, заданная соотношением $\left.(10)\right)$ и вид функции $\Gamma(x)$, то коэффициент затухания $\bar{\Gamma}$ определится соотношением:

$$
\bar{\Gamma}=\int_{-\infty}^{\infty} d x \Gamma(x) \rho_{1}^{(\mathrm{eq})}(x) .
$$

На втором временном интервале функции $n_{1}(t)$ и $x(t)$ существенно зависят от коэффициента диффузии (диффузионная стадия движения). Для параболической потенциальной энергии $U_{1}(x)$ и функции $\Gamma(x)$, задаваемой соотношением (9), вычисление интеграла в (11) приводит к следующему выражению для коэффициента затухания $\bar{\Gamma}$ :

$$
\begin{gathered}
\bar{\Gamma}=\gamma+\frac{1}{2} \tilde{\gamma}\left[\operatorname{erfc}(z)+(z \sqrt{\pi})^{-1}\left(1-e^{-z^{2}}\right)\right] \underset{z \gg 1}{\underset{z}{\approx}} \underset{\gamma}{\approx} \gamma+\frac{1}{2 z \sqrt{\pi}} \tilde{\gamma}\left(1-\frac{1}{2 z^{2}} e^{-z^{2}}\right), \\
z=\sqrt{\kappa a^{2} /(2 D)}=\sqrt{k a^{2} / 2 k_{B} T}
\end{gathered}
$$

$(\operatorname{erfc}(z)$ - дополнительная функция ошибок). Чем выше температура (больше значения параметра $D / a^{2}$ ), тем больше значения $\bar{\Gamma}$ и меньше длительность флуоресценции. Длительности спадания функций на втором участке, рассчитанные по формуле (12), совпадают с полученными из численных зависимостей (рис.3) и хорошо согласуются со спектроскопическими данными [24]. Подчеркнем, что зависимость обсуждаемых величин от температуры появляется исключительно из-за координатной зависимости константы скорости распада $\Gamma(x)$. Как отмечалось выше, если $\Gamma(x)=$ const, то характеристики возвратнопоступательного движения в параболических потенциалах не зависят от температуры в полном согласии с нашими прежними результатами [22].

Таким образом, в настоящей работе механизм функционирования управляемых светом возвратнопоступательных молекулярных машин (light-driven molecular machines) схематически рассмотрен на примере броуновского фотомотора. Он описан с помощью потенциальных энергий частицы в основном и возбужденном состояниях, а также координатной зависимости константы скорости распада возбужденного состояния. Эта модель имеет общий характер, единообразно описывая действие трансляционных, вращательных и возвратно-поступательных молекулярных машин.
При анализе возвратно-поступательных броуновских моторов их потенциальные энергии играют роль удерживающих потенциалов. Использование параболического приближения для этих потенциалов позволило получить самосогласованное описание в терминах не зависящей от температуры средней координаты частицы. Если период следования сверхкоротких лазерных импульсов меньше времени релаксации рассматриваемой системы, то частица не успевает возвращаться в начальное состояние. В таком случае возвратно-поступательное движение характеризуется переходной областью, в которой средняя координата частицы $x(t)$ претерпевает колебания между начальным значением и областью установившихся значений, где функция $x(t)$ становится периодической. Рассматриваемые процессы могут быть описаны аналитически, если константа скорости распада возбужденного состояния частицы не зависит от ее координаты. Средняя скорость возвратно-поступательного движения в установившемся режиме оказывается немонотонной функцией периода следования лазерных импульсов. Положение максимума этой функции задает оптимальный режим работы рассматриваемой молекулярной машины.

Описание возвратно-поступательного движения конкретизировано для молекулярной машины типа "гость-хозяин", в которой молекула красителя полностью втягивается в полость кавитанда при возбуждении лазерным импульсом и возвращается в начальное частично втянутое положение после дезактивации возбужденного состояния. Как обнаружено ранее [26], флуоресценция фотовозбужденной молекулы красителя тем продолжительнее, чем больше она втянута в кавитанд. Наблюдаемый эффект объясняется тем, что при полном втягивании молекулы красителя в кавитанд она не может релаксировать из возбужденного состояния в состояние ТICT, гасящее флуоресценцию. Данный эффект количественно описывается константой скорости распада возбужденного состояния, которая является убывающей функцией координаты молекулы красителя.

Построенная теория учитывает оптикомеханическую связь временных зависимостей заселенности возбужденного состояния частицы и ее координаты и тем самым объясняет известный экспериментальный факт - существенное увеличение времени жизни флуоресценции молекулы, полностью втянутой в кавитанд [24]. Получены общие соотношения, позволяющие выражать коэффициент затухания (обратную длительность флуоресценции) через потенциальный профиль возбужденного 
состояния, координатную зависимость константы скорости дезактивации системы и параметры модели.

Авторы благодарны Н. Х. Петрову за плодотворное обсуждение работы.

Работа выполнена в рамках Государственного задания 45.22 (регистрационный номер AАAА-А18-118012390045-2) и поддержана Российским фондом фундаментальных исследований (проекты 20-57-00007_Bel_а и 21-57-52006_MNT_а) и Белорусским республиканским фондом фундаментальных исследований (проект Ф20Р-032).

1. P. Hänggi and F. Marchesoni, Rev. Mod. Phys. 81, 387 (2009).

2. O. Kedem, B. Lau, M. A. Ratner, and E. A. Weiss, Proc. Natl. Acad. Sci. USA 114, 8698 (2017).

3. Ю. В. Гуляев, А. С. Бугаев, В. М. Розенбаум, Л.И. Трахтенберг, УФН 190, 337 (2020) [Phys.-Uspekhi 63, 311 (2020)].

4. J.A. Fornés, Principles of Brownian and Molecular Motors, Springer, Cham (2021).

5. P. Reimann, Phys. Rev. Lett. 86, 4992 (2001).

6. В. М. Розенбаум, И. В. Шапочкина, Ё. Тераниши, Л. И. Трахтенберг, Письма в ЖЭТФ 107, 525 (2018) [JETP Lett. 107, 506 (2018)].

7. M. L. Dekhtyar, A. A. Ishchenko, and V. M. Rozenbaum, J. Phys. Chem. B 110, 20111 (2006).

8. S. Saha and J. F. Stoddart, Chem. Soc. Rev. 36, 77 (2007).

9. Y.B. Zheng, H. Qingzhen, Y.-W. Yang, B. Kiraly, I.-K. Chiang, and T. J. Huang, J. Nanophotonics 4, 042501 (2010).

10. V.M. Rozenbaum, M.L. Dekhtyar, S.H. Lin, and L. I. Trakhtenberg, J. Chem. Phys. 145, 064110 (2016).

11. R. Augulis, M. Klok, B.L. Feringa, and
P.H. M. van Loosdrecht, Phys. Status Solidi C 6, 181 (2009).

12. В. М. Розенбаум, Письма в ЖЭТФ 88, 391 (2008) [JETP Lett. 88, 342 (2008)].

13. В. М. Розенбаум, И. В. Шапочкина, Л. И. Трахтенберг, УФН 189, 529 (2019) [Phys.-Uspekhi 62, 496 (2019)].

14. P. Reimann, Phys. Rep. 361, 57 (2002).

15. T. Hugel, N.B. Holland, A. Cattani, L. Moroder, M. Seitz, and H. E. Gaub, Science 296, 1103 (2002).

16. A. Mogilner, M. Mangel, and R. J. Baskin, Phys. Lett. A 237, 297 (1998).

17. H. C. Fogedby, R. Metzler, and A. Svane, Phys. Rev. E 70, 021905 (2004).

18. Yu. A. Makhnovskii, V.M. Rozenbaum, D.-Y. Yang, S. H. Lin, and T. Y. Tsong, Eur. Phys. J. B 52, 501 (2006).

19. А. А. Иванов, М. В. Алфимов, А. М. Желтиков, УФН 174, 743 (2004) [Phys.-Uspekhi 47, 687 (2004)].

20. V. V. Yakovlev, A. A. Ivanov, and V. Shcheslavskiy, Appl. Phys. B 74, 145 (2002).

21. Л.Д. Ландау, Е. М. Лифшиц, Квантовая механика. Нерелятивистская теория, Наука, М. (1974).

22. Yu.A. Makhnovskii, V.M. Rozenbaum, S.-Y. Sheu, D.-Y. Yang, L. I. Trakhtenberg, and S. H. Lin, J. Chem. Phys. 140, 214108 (2014).

23. A. I. Vedernikov, N.A. Lobova, L. G. Kuz'mina, J. A. K. Howard, Yu. A. Strelenko, M. V. Alfimov, and S. P. Gromov, J. Mol. Struct. 989, 114 (2011).

24. N. Kh. Petrov, D.A. Ivanov, Yu.A. Shandarov, I. V. Kryukov, A.A. Ivanov, M. V. Alfimov, N.A. Lobova, and S.P. Gromov, Chem. Phys. Lett. 647, 157 (2016).

25. Z. R. Grabowski, K. Rotkiewicz, and W. Rettig, Chem. Rev. 103, 3899 (2003).

26. Z. Li, S. Sun, F. Liu, Y. Pang, J. Fan, F. Song, and X. Peng, Dyes Pigm. 93, 1401 (2012). 